\title{
An investigation into the vitamin B1 and B2 content of a popular UK ready meal.
}

\author{
Robyn Rhule-Samuel, Pryank Patel and Xenofon Tzounis \\ University of Hertfordshire, Hatfield, United Kingdom
}

\section{Abstract}

Ready meals (RMs) are a convenient meal option, and it is assumed that they can be part of a balanced diet. Research has shown that energy, fat and salt content of RMs are "nutritionally chaotic"(Celnik et al., 2012), with many meals being significantly under- or overestimated in the labelling.

However, to our knowledge, no research has analysed the nutritional content of RMs in relation to labile water-soluble vitamins undergoing food processing, such as vitamin B1 or B2. Accurate estimations of certain water-soluble vitamins is important to understand whether RMs contribute sufficient amounts of these essential vitamins to the diet to meet the nutrient requirements for those who consistently consume RMs.

The 'sausage and mash' range of RM is one of the most popular RMs eaten by meals-on-wheels users and purchased in supermarkets. The aim of this study is to compare process-labile vitamin content; vitamin B1 and B2, in sausage and mash RMs, and carry out price comparison between meals.

Sausage and mash RMs from five RM suppliers were analysed in duplicate. Following derivatisation of Vitamin B1, both vitamin B1 and B2 were analysed with fluorescent detection using HPLC. All food samples were tested in triplicate. Kruskal-Wallis test was carried out to compare vitamin content between RMs from different RM providers. Pearsons correlation tested relationships between meal composition and RM price.

RMs significantly differed between providers where vitamin $\mathrm{B} 1\left(\mathrm{x}^{2}=8.073, \mathrm{p}<0.05\right)$ and $\mathrm{B} 2\left(\mathrm{x}^{2}=7.964, \mathrm{p}<0.05\right)$ content was between $1.05-3.73 \mathrm{mg} /$ serving and $0.033-0.098 \mathrm{mg} / \mathrm{serving}$, respectively. The price of the RMs ranged between $£ 2.00-£ 3.50$. Analysis showed that cheaper meals had a higher thiamine content $(r=-0.675, p<0.001)$, which could be due to the presence of green peas; a source of thiamine, in one of the cheaper RMs. Results showed that the percentage $(\%)$ of meat/serving was higher in the more expensive meals $(r=0.603, p<0.001)$, and the cheaper meals had a higher percentage of mashed potatoes $(r=-0.837$, $\mathrm{p}<0.001)$.

This study shows that there are differences in vitamin B1 and B2 between RM providers. This may mean that consumers are not aware of which meals may be the most nutritionally adequate. Price of meal has an impact on composition and therefore nutrient density. Future studies should analyse other process labile essential vitamins such as vitamin C and other B-group vitamins to establish accurate estimations of water-soluble vitamin content in RMs.

\section{Conflict of Interest}

There is no conflict of interest 